\title{
The Geometric Derivation of the Transformation of Time and Position Coordinates in STE
}

\author{
Karol Szostek ${ }^{1}$, Roman Szostek ${ }^{2}$ \\ ${ }^{1}$ Rzeszow University of Technology, Dept of Thermodynamics and Fluid Mechanics, Rzeszow, Poland \\ ${ }^{2}$ Rzeszow University of Technology, Department of Quantitative Methods, Rzeszow, Poland
}

\begin{abstract}
It is commonly thought that the Michelson-Morley experiment from 1887 [1] and Kennedy-Thorndike experiment from 1932 [2] demonstrated that the ether does not exist and that the velocity of light in vacuum is absolutely constant. The analysis of this experiment led to the creation of the special theory of relativity (STR). In this article, a different transformation of time and position than the Lorentz transformation is derived on the basis of the geometric analysis of the Michelson-Morley experiment. The transformation is derived based on the assumption that the universal frame of reference, called the ether, exists. Our concept states that the ether is not a substance with physical properties, as it was understood in the past, but a frame of reference in which the velocity of light is constant in every direction. In inertial frames of reference moving in the ether, the velocity of light may be different. The obtained transformations are transformations from the ether to the inertial frame and from the inertial frame to the ether. Based on the new transformation, the Special Theory of Ether (STE) was created [4], [5].
\end{abstract}

Keywords: Special Theory of Ether, Special Theory of Relativity, coordinate and time transformation

\section{Introduction}

In the article, the transformations from the inertial system to the ether and from the ether to the inertial system was derived by the geometric method shown in [3]. An explanation of the results of the MichelsonMorley and Kennedy-Thorndike experiments, assuming the existence of the ether, in which the velocity of light is constant, is presented. In inertial frames of reference moving in the ether, the velocity of light may be different.

The velocity of light in one direction has never been accurately measured. In all accurate laboratory experiments, as in the Michelson-Morley experiment, only the average velocity of light, travelling on a closed trajectory, was measured. In these experiments, light always comes back to the source point.

The transformation ether-system (29) derived in this article by the geometric method was already derived in article [6] and [7] by other method. In that case, the author obtained this transformation thanks to the synchronization of clocks in inertial frames by the external method. Works [4] and [5] demonstrated that although the transformation from work [6] has the same form as the transformation derived in this article, it has different meaning. The transformation obtained in work [6] is equivalent to the Lorentz transformation. It is only the Lorentz transformation differently written down after a change in the manner of time measurement in the inertial frame of reference. The transformation derived in this article has a different meaning than the Lorentz transformation. The transformation obtained here binds together coordinates of position which are next to each other, while the Lorentz transformation converts the coordinates of position to past and future [4], [5].

\section{The Assumptions}

In the presented analysis of the Michelson-Morley experiment, the following assumptions are adopted:

$>$ There is a frame of reference with respect to which the velocity of light in vacuum is the same in every direction. This universal frame of reference is called the ether.

$>$ The average velocity of light on its way to and back is for every observer independent of the direction of light propagation. This results from the Michelson-Morley experiment.

$>$ The average velocity of light on its way to and back does not depend on the velocity of the observer in relation to the universal frame of reference (the ether). This results from the Kennedy-Thorndike experiment.

$>$ In the direction perpendicular to the direction of the velocity of the body, moving in relation to the ether, there is no contraction of its length.

$>$ The transformation ether-system is linear.

$>$ Between the ether and the inertial frame, there is some symmetry in the following form: 


$$
\left.\frac{d x}{d t^{\prime}}\right|_{\frac{d x^{\prime}}{d t^{\prime}}=0}=\left.\frac{d x^{\prime}}{d t}\right|_{\frac{d x}{d t}=0}
$$

This assumption means that the differential of the body position in the ether, resting in the inertial frame, determined after the time measured in the inertial frame is the same as the differential of the body position in the inertial frame, resting in the ether, determined after the time measured in the ether.

The transformation derivation presented in this article differs from the derivation by the geometric method of the Lorentz transformation which is the basis for the STR. In the derivation of the Lorentz transformation, it is assumed that the reverse transformation has the same form as the original transformation. Such an assumption stems from the belief that all inertial frames are equivalent. In the derivation presented in this article, we do not assume what form the whole reverse transformation has. We only adopt the assumption concerning one coefficient in the reverse transformation, occurring at $t$ in the equation concerning position coordinate (16) (assumption VI).

In works [4], [5], identical transformations were derived as in this article, but without adopting assumption VI. For this, it was necessary to conduct the full analysis of the Michelson-Morley experiment in which also the second stream of light, parallel to velocity $v$, is taken into account.

Assumptions concerning the velocity of light adopted in this article are also weaker than those adopted in the STR. In the STR, it is assumed that the velocity of light is absolutely constant, despite the fact that it has not been proven by any experiment. In this article, the assumption resulting from experiments is adopted, i.e. the average velocity of light on the way to the mirror and back is constant (assumption II and III). In the presented considerations, the velocity of light by assumption is constant only in one highlighted frame of reference - the ether (assumption I). Assumptions IV and V are identical to those on which the STR is based.

\section{The Geometrical Derivation of the Transformation}

We analyze the results of the Michelson-Morley experiment, as shown in Figure 1. The inertial system $U^{\prime}$ moves at a relative velocity $v$ to the inertial system $U$, associated with the ether, parallel to the axis $x$. Systems have horizontal axes facing opposite directions, to keep symmetry between them.

At the moment when origins of systems overlap, clocks in both systems are synchronized. Clocks in system $U$ related to the ether are synchronized by the internal method [6]. Clocks in system $U^{\prime}$ are synchronized by the external method in such a manner that if the clock of system $U$ indicates time $t=0$, then the clock of system $U^{\prime}$ next to it is also reset, that is $t^{\prime}=0$.

At the time when the origins of systems overlap, a signal of light is sent inside the system $U^{\prime}$, from the origin of the system, parallel to the $y^{\prime}$ axis. This signal after traveling the distance $D^{\prime}$ is reflected from the mirror and returns to the origin of the coordinate system (part a of Figure 1). The same signal is observed from the system $U$, wherein, due to the relative motion of the systems, light moves along the arms of the triangle (part b). System $U$ is the universal frame of reference in which light has always constant velocity $c$ (assumption I). Considerations concern the flow of light in vacuum.

In accordance with conclusions resulting from the Michelson-Morley experiment it has been assumed that the average velocity of light $c_{p}$ on the way to the mirror and back in system $U^{\prime}$ is the same in every direction, in particular in the parallel direction to the axis $y^{\prime}$ (assumption II). It has also been assumed that the average velocity of light $c_{p}$ on the way to the mirror and back does not depend on the velocity of an observer in relation to the ether (assumption III).

From assumption II and III it follows that the average velocity of light $c_{s}$ in the inertial frame of reference is the same as the velocity of light $c$ in the ether. It is sufficient to note that the light signal parallel to the axis $y^{\prime}$ has in system $U^{\prime}$ the same average velocity $c_{p}$ also when system $U^{\prime}$ does not move in relation to system $U(v=0)$. Because then velocity $c_{p}$ is precisely the same as velocity $c$, therefore for every velocity $v c_{p}=c$ is true.

Formally, it can be written down as follows. If we allow that the average velocity of light $c_{p}$ in the system $U^{\prime}$ is a function of the velocity of light $c$ in the system $U$, i.e. the ether dependent on the velocity $v$, we can write

$$
c_{p}=f(v) c
$$

From measurement, we know that the average velocity of light is the same for different velocities of the Earth relative to the ether, so $f\left(v_{1}\right)=f\left(v_{2}\right)$. Since $f(0)=1$, therefore $f(v)=1$ for every velocity $v$. It follows that $c=c_{p}$.

If the velocity of light in relation to the ether is the same in every direction and equal to $c$, then due to the constant value of velocity $v$, a triangle along which light moves in system $U$ is isosceles (Figure 1). 

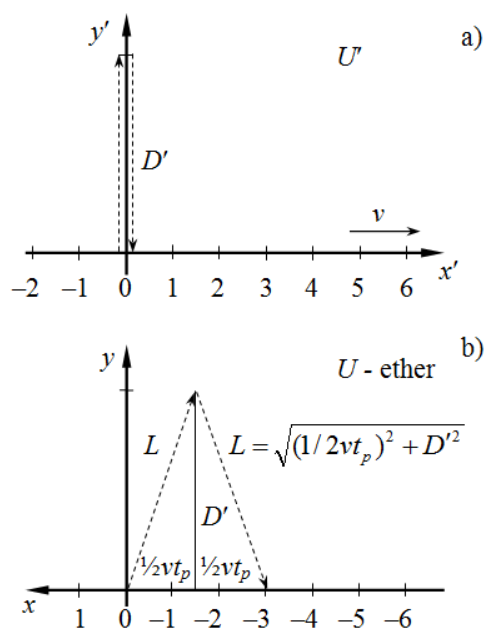

Fig. 1. The light flow distances in the inertial frame of reference and in the ether

a) The light flow in the system $U^{\prime}$ parallel to the axis $y^{\prime}$ b) The light flow seen from the ether $U$

If we denote the flow time of light measured in the system $U^{\prime}$ by $t_{p}{ }^{\prime}$ and denote the flow time of light measured in the system $U$ by $t_{p}$, we obtain

Hence we have

$$
c_{p}=\frac{2 D^{\prime}}{t_{p}^{\prime}}=c=\frac{2 L}{t_{p}}
$$

$$
\begin{aligned}
D^{\prime} & =\frac{1}{2} t_{p}^{\prime} c \\
L & =\frac{1}{2} t_{p} c
\end{aligned}
$$

We assume that the distance $D^{\prime}$, which is perpendicular to the velocity $v$ is the same for observers from both systems (assumption IV). Therefore, in Figure 1, there is the same length $D^{\prime}$ in part a and part b.

At the time when light is reflected from the mirror, the origin of the system $U^{\prime}$ is in the system $U$ in position $1 / 2 v t_{p}$. Length $L$ of the distance light travels in the system $U$ can be written as

$$
L=\sqrt{\left(\frac{1}{2} v t_{p}\right)^{2}+D^{\prime 2}}
$$

Having squared the above equation and applied (4) and (5), we obtain

$$
\begin{gathered}
\left(\frac{1}{2} t_{p} c\right)^{2}=\left(\frac{1}{2} v t_{p}\right)^{2}+\left(\frac{1}{2} t_{p}^{\prime} c\right)^{2} \\
t_{p}^{2}\left(c^{2}-v^{2}\right)=\left(t_{p}^{\prime} c\right)^{2} \\
t_{p}=t_{p}^{\prime} \frac{1}{\sqrt{1-(v / c)^{2}}}
\end{gathered}
$$

The above relation describes only times $t_{p}$ and $t_{p}{ }^{\prime}$ that involve the full light flow to the mirror and back. However, if we assume that the length $D^{\prime}$ can be chosen so that $t_{p}{ }^{\prime}$ is any time, so the relationship (9) is true for any time. So we have

$$
t=t^{\prime} \frac{1}{\sqrt{1-(v / c)^{2}}}
$$

The velocity $v$ is the velocity of the system $U^{\prime}$ that is seen from $U$ i.e. the ether. If a position of the origin of the system $U^{\prime}\left(x^{\prime}=0\right)$ in the system $U$ is denoted by $x$, then

$$
v=-\frac{x}{t}
$$


Having determined the coordinate $x$ from equation (11), and applied (10), we obtain a transformation for the coordinate $x^{\prime}=0$

$$
x=-v t=-v t^{\prime} \frac{1}{\sqrt{1-(v / c)^{2}}} \quad \text { for } \quad x^{\prime}=0
$$

This relationship expresses the position of the origin of the inertial system $U^{\prime}$ i.e. $\left(x^{\prime}=0\right)$ that is at time $t^{\prime}$ measured from the system $U$.

The transformation between the inertial system and the ether should be linear (assumption V), because if a body moving with uniform motion in the system $U^{\prime}$, it should be assumed that in the system $U$ it also moves with uniform motion. On the basis of (10) and (12), we have the transformation from the system $U^{\prime}$ to $U$ in the form of

$$
\begin{gathered}
t=t^{\prime} \frac{1}{\sqrt{1-(v / c)^{2}}}+a^{\prime} x^{\prime} \\
x=-v t^{\prime} \frac{1}{\sqrt{1-(v / c)^{2}}}+b x^{\prime}
\end{gathered}
$$

In above equations, there are two unknown parameters $a^{\prime}$ and $b$. At this stage we do not know what the values of these parameters are.

These above equations are the transformation of time and position from the system $U^{\prime}$ to the system $U$. We assume that the transformation from the system $U$, to the system $U^{\prime}$ has the form

$$
\begin{gathered}
t^{\prime}=t \sqrt{1-(v / c)^{2}}+a^{\prime \prime} x \\
x^{\prime}=-v t \frac{1}{\sqrt{1-(v / c)^{2}}}+\frac{1}{b} x
\end{gathered}
$$

A coefficient before $t$ in equation (15) is the reciprocal of the ratio of the coefficient before $t^{\prime}$ in equation (13). Hence, if in the system $U$ more time than in the system $U^{\prime}$ elapsed, then in the system $U^{\prime}(13)$ accordingly less time than in the system $U(15)$ must elapse.

It is similarly with the coefficient $1 / b$ placed in equation (16) before $x$. It is the reciprocal of the coefficient $b$ occurring in equation (14) before $x^{\prime}$. If in the system $U^{\prime}$, the value $x^{\prime}$ will be changed, according to (14) in the system $U$, the value $x$ will change $b$ times more. In the other way, the situation must be reversed. If in the system $U$, the value $x^{\prime}$ will be changed, then in the system $U^{\prime}$, the value $x^{\prime}$ will change $b$ times less.

The coefficient before $t$ in equation (16) is the same as the coefficient before $t^{\prime}$ in equation (14). These coefficients have the same sign, due to opposite directions of $X$ and $X^{\prime}$ axes of systems $U$ and $U^{\prime}$. If with passage of time $t^{\prime}$, the coordinate $x^{\prime}$ decreases in the system $U$, then, similarly, with the passage of time $t$, the coordinate $x$ decreases in the system $U^{\prime}$. The same value of these coefficients results from assumption VI. To coefficients $a^{\prime}$ and $a^{\prime \prime}$ we do not accept any assumptions.

In a particular case, if we determine the value of coordinate $x=0$ transformations (13)- (16) take the form

$$
\begin{gathered}
t=t^{\prime} \frac{1}{\sqrt{1-(v / c)^{2}}}+a^{\prime} x^{\prime} \\
0=-v t^{\prime} \frac{1}{\sqrt{1-(v / c)^{2}}}+b x^{\prime} \\
t^{\prime}=t \sqrt{1-(v / c)^{2}} \\
x^{\prime}=-v t \frac{1}{\sqrt{1-(v / c)^{2}}}
\end{gathered}
$$

Having eliminated $t^{\prime}$ and $x^{\prime}$, we obtain

$$
t=t-a^{\prime} v t \frac{1}{\sqrt{1-(v / c)^{2}}}
$$




$$
0=-v t-b v t \frac{1}{\sqrt{1-(v / c)^{2}}}
$$

From dependence (21) we obtain that $a^{\prime}=0$. In an analogous manner, if we assume the value of $x^{\prime}=0$, it can be demonstrated that $a^{\prime \prime}=0$. From dependence (22) we obtain

$$
\begin{gathered}
0=-v t-b v t \frac{1}{\sqrt{1-(v / c)^{2}}} \\
b=-\sqrt{1-(v / c)^{2}}
\end{gathered}
$$

The transformation from the inertial system to the ether is in the form

$$
\begin{gathered}
t=\frac{1}{\sqrt{1-(v / c)^{2}}} t^{\prime} \\
x=-\frac{1}{\sqrt{1-(v / c)^{2}}} v t^{\prime}-\sqrt{1-(v / c)^{2}} \cdot x^{\prime}
\end{gathered}
$$

while the transformation from the ether to the inertial system is in the form

$$
\begin{gathered}
t^{\prime}=\sqrt{1-(v / c)^{2}} \cdot t \\
x^{\prime}=\frac{1}{\sqrt{1-(v / c)^{2}}}(-v t-x)
\end{gathered}
$$

If you change the direction of the horizontal axis in the system $U$, i.e. the ether, then the value of $x$ must be replaced in the above transformations by $-x$. Then, we obtain the transformation, from any inertial system to the ether, in the form

$$
\left\{\begin{array}{l}
t=\frac{1}{\sqrt{1-(v / c)^{2}}} t^{\prime} \\
x=\frac{1}{\sqrt{1-(v / c)^{2}}} v t^{\prime}+\sqrt{1-(v / c)^{2}} \cdot x^{\prime}
\end{array}\right.
$$

and the inverse transformation from the ether to any inertial system

$$
\left\{\begin{array}{l}
t^{\prime}=\sqrt{1-(v / c)^{2}} \cdot t \\
x^{\prime}=\frac{1}{\sqrt{1-(v / c)^{2}}}(-v t+x)
\end{array}\right.
$$

We assume that

\section{Relative Velocities Between Systems}

$$
\delta(v)=1-(v / c)^{2}
$$

Consider the three systems: $U$ associated with the ether and two inertial systems $U_{1}$ and $U_{2}$. Systems have been presented at two time instants $t=0$ and $t=T$. On the axis of the ether, two coordinates $A$ and $B$, which correspond to places in the ether, where origins of systems $U_{1}$ and $U_{2}$ will be at time $T$, are marked. The system $U_{1}$ has velocity $v_{1}=A / T$, while the system $U_{2}$ has velocity $v_{2}=B / T$ relative to the ether (Figure 2 ). elapsed

Times in inertial systems $t_{1}$ and $t_{2}$ are calculated from transformation (29), when in the ether time $T$ had

$$
\begin{aligned}
& t_{1}=T \sqrt{\delta\left(v_{1}\right)} \\
& t_{2}=T \sqrt{\delta\left(v_{2}\right)}
\end{aligned}
$$

The transformation from the ether to the system is used to convert coordinates. Coordinate $B$ is converted to the system $U_{1}$ and is marked as $B_{1}$, coordinate $A$ is converted to the system $U_{2}$ and is marked as $A_{2}$. Thus, on the basis of (29) we can write 


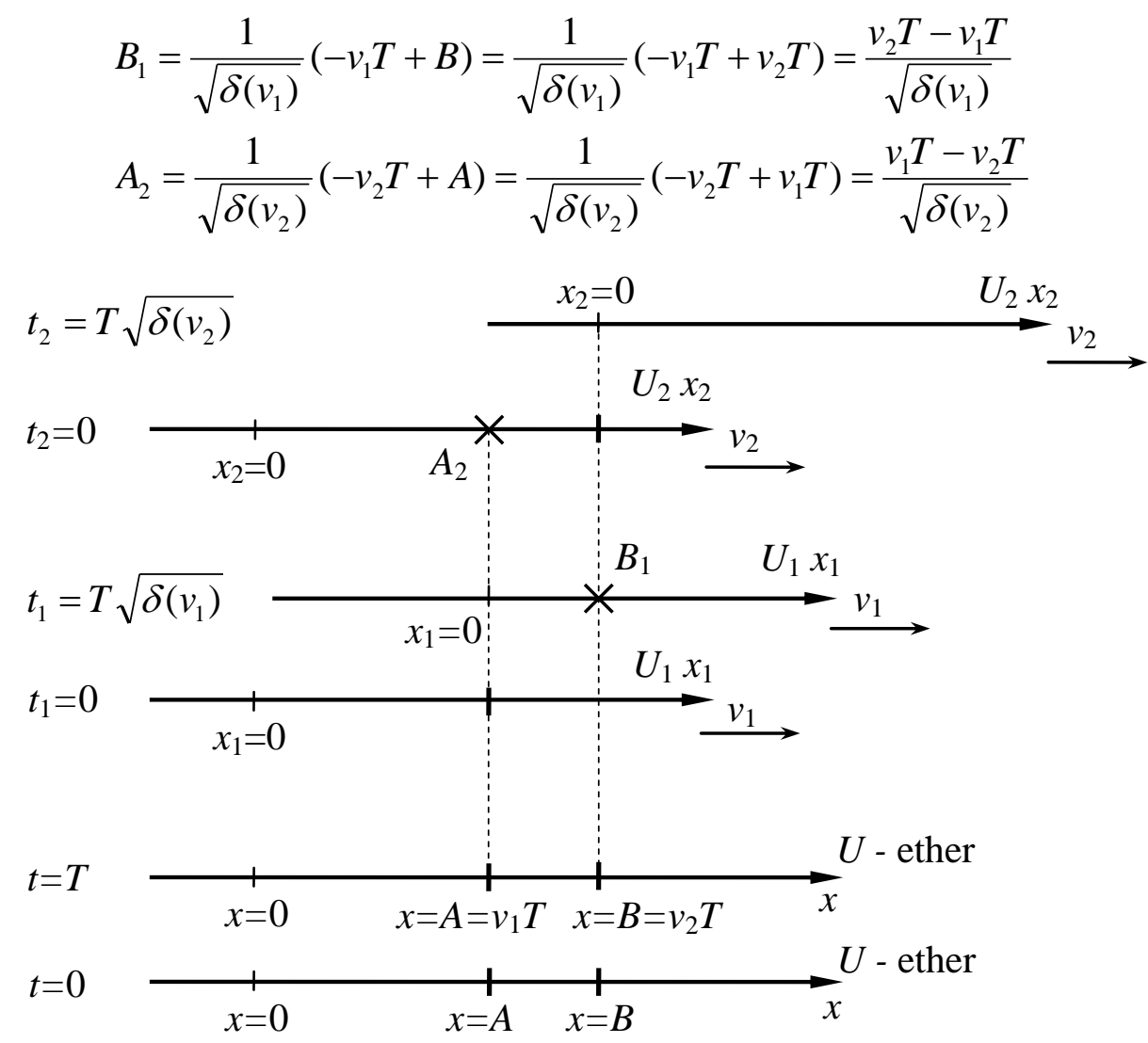

Fig. 2. The inertial systems $U_{1}, U_{2}$ moving in the ether at velocities $v_{1}, v_{2}$

Coordinate of $B_{1}$ corresponds to the distance that the system $U_{2}$ travelled in relation to the system $U_{1}$. Coordinate $A_{2}$ corresponds to the distance that the system $U_{1}$ travelled in relation to the system $U_{2}$. The velocity $v_{2 / 1}$ of the system $U_{2}$ relative to the system $U_{1}$ is $B_{1} / t_{1}$. The velocity $v_{1 / 2}$ of the system $U_{1}$ relative to the system $U_{2}$ is $A_{2} / t_{2}$. Having divided equations (32) by equations (31), we received the equation that describes relative velocities

$$
\begin{aligned}
& v_{2 / 1}=B_{1} / t_{1}=\left(v_{2}-v_{1}\right) \frac{1}{\delta\left(v_{1}\right)}=\frac{v_{2}-v_{1}}{1-\left(v_{1} / c\right)^{2}} \\
& v_{1 / 2}=A_{2} / t_{2}=\left(v_{1}-v_{2}\right) \frac{1}{\delta\left(v_{2}\right)}=\frac{v_{1}-v_{2}}{1-\left(v_{2} / c\right)^{2}}
\end{aligned}
$$

Having divided (34) by (33), we obtain a dependence binding relative velocities

$$
-\frac{v_{1 / 2}}{v_{2 / 1}}=\frac{\delta\left(v_{1}\right)}{\delta\left(v_{2}\right)}=\frac{1-\left(v_{1} / c\right)^{2}}{1-\left(v_{2} / c\right)^{2}}=\frac{c^{2}-v_{1}^{2}}{c^{2}-v_{2}^{2}}
$$

\section{The Velocity of Light in the Inertial Reference System}

Now we calculate the velocity of light in any inertial system $U_{1}$. Consider three inertial reference systems $U_{1}, U_{2}$ and $U_{3}$ moving in the ether in parallel to the $x$ axis, Figure 3. Systems $U_{2}$ and $U_{3}$ are associated with light, but move in opposite directions. Therefore, their velocities in the ether are $v_{2}=c$ and $v_{3}=-c$. The system $U_{1}$ moves in the ether at the velocity $v_{1} \geq 0$. From equation (33), the velocity of light. measured in the system $U_{1}$, can be calculated

and

$$
c_{p 1}=v_{2 / 1}=\frac{c-v_{1}}{1-\left(v_{1} / c\right)^{2}}=\frac{c^{2}\left(c-v_{1}\right)}{c^{2}-v_{1}^{2}}=\frac{c^{2}\left(c-v_{1}\right)}{\left(c+v_{1}\right)\left(c-v_{1}\right)}=\frac{c^{2}}{c+v_{1}} \leq c
$$

$$
c_{p 2}=v_{3 / 1}=\frac{-c-v_{1}}{1-\left(v_{1} / c\right)^{2}}=-\frac{c^{2}\left(c+v_{1}\right)}{c^{2}-v_{1}^{2}}=-\frac{c^{2}\left(c+v_{1}\right)}{\left(c+v_{1}\right)\left(c-v_{1}\right)}=-\frac{c^{2}}{c-v_{1}} \leq-c
$$




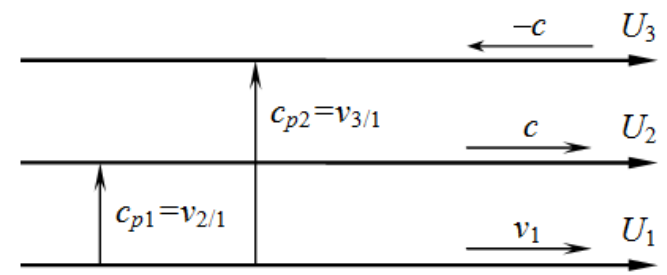

Fig. 3. The velocity of light in one direction

When light travels in the ether in the same direction as the inertial system $U_{1}$, then the velocity of light in the system is expressed by equation (36). When light travels in the ether in the direction opposite to the inertial system $U_{1}$, then the velocity of light in the system is expressed by equation (37). Charts of the velocity as a function of $v_{1}$ are shown in Figure 4. Lines in the graph determine the velocity range that can be observed from the inertial system $U_{1}$.

For boundary velocities $v_{1}$ of the system we obtain

$$
\begin{gathered}
\lim _{v_{1} \rightarrow c} c_{p 1}=\frac{c^{2}}{c+v_{1}}=\frac{c^{2}}{2 c}=\frac{1}{2} c \\
\lim _{v_{1} \rightarrow c} c_{p 2}=\lim _{v_{1} \rightarrow c}\left(-\frac{c^{2}}{c-v_{1}}\right)=-\frac{c^{2}}{c-c}=-\infty
\end{gathered}
$$

and

$$
\begin{gathered}
\lim _{v_{1} \rightarrow 0} c_{p 1}=\lim _{v_{1} \rightarrow 0} \frac{c^{2}}{c+v_{1}}=c \\
\lim _{v_{1} \rightarrow 0} c_{p 2}=\lim _{v_{1} \rightarrow 0}\left(-\frac{c^{2}}{c-v_{1}}\right)=-c
\end{gathered}
$$

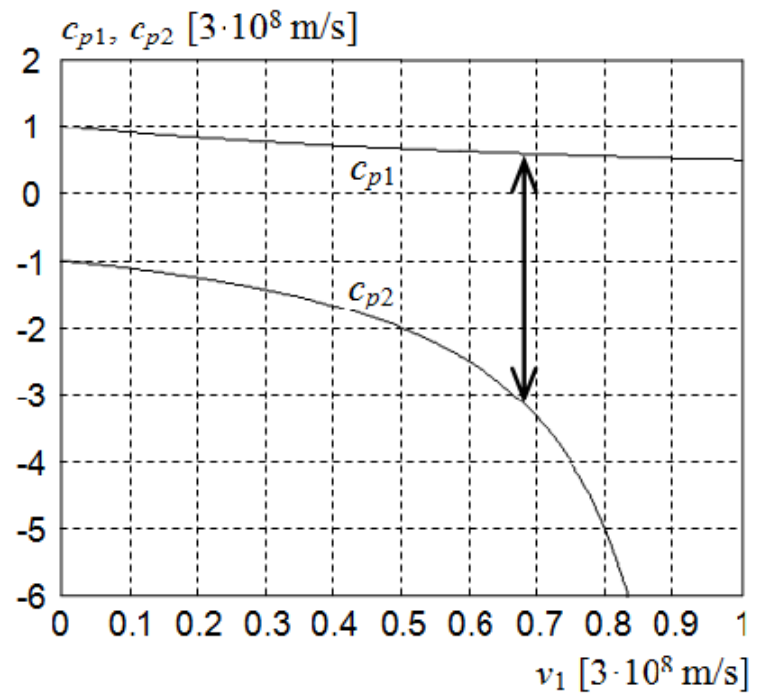

Fig. 4. The velocity of light in the inertial system for $v_{1} \geq 0$

It follows that if the system $U_{1}$ moves at velocity close to $c$, then light running in the same direction in relation to the system $U_{1}$ has velocity close to $1 / 2 c$. Light running in the opposite direction has infinite velocity to the system $U_{1}$. Thus, the velocity of light in relation to the inertial system can be very high, because the system clocks go more slowly than in the ether. The velocity of light in the ether is exactly $c$.

In the system $U_{1}$, let light run in parallel to velocity $v_{1}$ of the system $U_{1}$ relative to the ether. Just as in the Michelson-Morley experiment, light runs along the way $L$ over time $t^{\prime}$. At the end of the way, light is reflected in the mirror and goes back along the same way $L$ over time $t^{\prime \prime}$. Then, the average velocity of light can be described on the basis of (36) and (37) and is equal to 


$$
c_{s r}=\frac{2 L}{t^{\prime}+t^{\prime \prime}}=\frac{2 L}{\frac{L}{c_{p 1}}+\frac{L}{c_{p 2}}}=\frac{2 L}{\frac{L}{\frac{c^{2}}{c+v_{1}}}+\frac{L}{\frac{c^{2}}{c-v_{1}}}}=\frac{2}{\frac{c+v_{1}}{c^{2}}+\frac{c-v_{1}}{c^{2}}}=\frac{2}{\frac{2 c}{c^{2}}}=c
$$

This velocity agrees with the results of the Michelson-Morley experiment, which shows that the average velocity of light is constant and is equal to $c$ (the average velocity, not instantaneous). We have shown that the Michelson-Morley experiment does not imply that the current velocity of light is constant in every direction.

Velocities expressed in dependencies (36) and (37) are different. The first one refers to the direction compatible with velocity $v_{1}$, and the other concerns the direction opposite to velocity $v_{1}$. However, the average velocity of light is constant and is equal to $c$.

In works [4], [5], a general formula for the velocity of light running in any direction is derived. For light moving in vacuum, it has the form of

$$
c_{\alpha}^{\prime}=\frac{c^{2}}{c+v \cos \alpha}
$$

For light moving in a material medium motionless in relation to the observer, it has the form of

$$
c_{s \alpha}^{\prime}=\frac{c^{2} c_{s}}{c^{2}+c_{s} v \cos \alpha}
$$

In these two dependencies, angle $\alpha$ is the angle, measured by the observer, between the vector of its velocity in relation to the ether and the vector of the velocity of light. Velocity $c_{s}$ is the velocity of light in the material medium motionless in relation to the ether seen by the observer motionless in relation to the ether.

Formulas (43) and (44) come down to formulas (36) and (37), if we substitute $c_{s}=c$ and $\alpha=0^{\circ}$ or $\alpha=180^{\circ}$. Formulas (43) and (44) also have the property presented in (42). It is sufficient to verify that for the velocity of light expressed by formula (44), the average velocity on the way to the mirror and back is equal to

$$
\begin{gathered}
c_{s r}^{\prime}=\frac{2 L}{t_{s \alpha}^{\prime}+t_{s(180-\alpha)}^{\prime}}=\frac{\frac{L L}{\frac{c^{2} c_{s}}{c^{2}+c_{s} v \cos \alpha}}+\frac{L}{\frac{c^{2} c_{s}}{c^{2}+c_{s} v \cos (180-\alpha)}}}{c_{s r}^{\prime}=\frac{2}{\frac{c^{2}+c_{s} v \cos \alpha}{c^{2} c_{s}}+\frac{c^{2}-c_{s} v \cos \alpha}{c^{2} c_{s}}}=\frac{2}{\frac{2 c^{2}}{c^{2} c_{s}}}=c_{s}}
\end{gathered}
$$

From dependence (46) it follows that $c_{s}$ is also the average velocity of light on the way to the mirror and back in the material medium motionless in relation to the observer. Despite the fact that the velocity of light expressed by formula (44) depends on angle $\alpha$ and velocity $v$, the average velocity of light on the way to the mirror and back is always constant and is equal to $c_{s}$.

\section{Conclusion}

Derived transformations (28)-(29) are consistent with the Michelson-Morley experiment. From the above transformations it follows that the measurement of the velocity of light in vacuum by means of the previously applied methods will always give the average value equal to $c$. This happens despite the fact that for the moving observer the velocity of light has a different value in different directions. The average velocity of light is always constant and independent of the velocity of the inertial frame of reference.

We demonstrated that the explanation of the results of the Michelson-Morley experiment and other experiments with light is possible, provided that the fact that the velocity of light is not constant for every observer is acceptable.

Admitting that the velocity of light may depend on the direction of its emission does not differentiate any direction in space. The velocity of light which is measured by the moving observer is significant here. It is the velocity at which the observer moves in relation to the universal frame of reference (the ether) that differentiates the characteristic direction in space, but only for this observer. For the observer motionless in relation to the universal frame of reference, the velocity of light is always constant and does not depend on the direction of its emission. If the observer moves in relation to the universal frame of reference, then from his perspective space is not symmetrical. The case of this observer will be similar to the case of the observer 
moving on water and measuring the velocity of the wave on water. Despite that the wave propagates on water at the constant velocity in every direction, from the perspective of the observer moving on water, the velocity of the wave will be different in different directions.

The Michelson-Morley experiment and Kennedy-Thorndike experiment were conducted many times by different teams. Also modified and improved versions of this experiment, like the experiment with sapphire crystals from 2015 [8], were conducted. Each of the experiments only confirmed that the average velocity of light is constant.

It follows from the conducted analysis that the explanation of the results of the Michelson-Morley experiment on the basis of the ether is possible. Stating that the Michelson-Morley experiment proved that the velocity of light is absolutely constant is untrue. Stating that the Michelson-Morley experiment proved that there is no ether in which light propagates and moves at a constant velocity is also untrue.

\section{Bibliography}

[1] Michelson Albert A., Morley Edward W., On the relative motion of the earth and the luminiferous ether. Am. J. Sci. 34, 333-345 (1887).

[2] Halliday David, Resnick Robert i Walker Jearl., Fundamentals of Physics, part 5, chapter 39, Wiley-VCH , 2004, ISBN 0-47142965-1.

[3] Szostek Karol, Szostek Roman, Special Theory of Ether (in English), Publishing house AMELIA, Rzeszow in Poland 2015, ISBN 978-83-63359-81-2 (www.ste.com.pl).

[4] Szostek Karol, Szostek Roman, Szczególna Teoria Eteru (in Polish), Wydawnictwo Amelia, Rzeszów w Polsce 2015, ISBN 97883-63359-77-5 (www.ste.com.pl).

[5] Tangherlini Frank R., The Velocity of Light in Uniformly Moving Frame, A Dissertation. Stanford University, 1958 (reprint in The Abraham Zelmanov Journal, Vol. 2, 2009, ISSN 1654-9163)

[6] Mansouri Reza, Sexl Roman U., A Test Theory of Special Relativity: I. Simultaneity and Clock Synchronization, General Relativity and Gravitation, Vol. 8, No. 7 (1977), pp. 497-513.

[7] Kennedy Roy J., Thorndike Edward M., Experimental Establishment of the Relativity of Time. „Physical Review”. 42 (3), s. 400418, 1932. DOI: 10.1103/PhysRev.42.400

[8] Nagel Moritz, Parker Stephen R., Kovalchuk Evgeny V., Stanwix Paul L., Hartnett John G., Ivanov Eugene N., Peters Achim, Tobar Michael E., Direct terrestrial test of Lorentz symmetry in electrodynamics to $10^{-18}$, Nature Communications 6 , Article number: 8174, 1 september 2015, DOI: 10.1038/ncomms9174. 